\title{
Dessein, dessin, design... Les tribulations d'Idea en régime de haut modernisme américain
}

\section{Nicole Dubreuil}

Volume 37, numéro 2, 2012

Idea in Art

L'idée dans l'art

URI : https://id.erudit.org/iderudit/1066725ar

DOI : https://doi.org/10.7202/1066725ar

\section{Aller au sommaire du numéro}

\section{Éditeur(s)}

UAAC-AAUC (University Art Association of Canada | Association d'art des universités du Canada)

\section{ISSN}

0315-9906 (imprimé)

1918-4778 (numérique)

\section{Découvrir la revue}

Citer cet article

Dubreuil, N. (2012). Dessein, dessin, design... Les tribulations d'Idea en régime de haut modernisme américain. RACAR : Revue d'art canadienne / Canadian Art Review, 37(2), 60-72. https://doi.org/10.7202/1066725ar

\section{Résumé de l'article}

This article examines the survival, in post-World War II American abstract painting and especially in the formalist critique interpreting and legitimizing it, of age-old debates concerning the primacy of thought over the mimetic representation of the world that have characterized occidental art theory since Plato. The history of these debates, which the Renaissance took up again and channeled in more aesthetic than metaphysical directions, has been masterfully summarized by Erwin Panofsky in his celebrated essay of 1924 dedicated to idea. One of the pivotal points of that theory as a whole is the relationship established between the plan or intention of the work (dessein) and the drawing or sketch (dessin) which gives it form. This article argues that modernist painting, although radically abstract and apparently devoted to the triumph of the colour field alone, paradoxically fails to reflect upon itself without recourse to a principle of internal articulation wherein subsist avatars of the old alliance between dessein and dessin. The radical transformation of art's context of production and reception, brought about by the instauration of modernity, introduces however a third term into the equation, design (de-sign), which expresses the fear of a definitive loss: that of the capacity of art to reflect upon the world and provide meaning.
Tous droits réservés (C) UAAC-AAUC (University Art Association of Canada | Association d'art des universités du Canada), 2012
Ce document est protégé par la loi sur le droit d'auteur. L’utilisation des services d'Érudit (y compris la reproduction) est assujettie à sa politique d'utilisation que vous pouvez consulter en ligne.

https://apropos.erudit.org/fr/usagers/politique-dutilisation/ 


\title{
Dessein, dessin, design... Les tribulations d'Idea en régime de haut modernisme américain
}

\author{
Nicole Dubreuil, Université de Montréal
}

\begin{abstract}
This article examines the survival, in post-World War II American abstract painting and especially in the formalist critique interpreting and legitimizing it, of age-old debates concerning the primacy of thought over the mimetic representation of the world that have characterized occidental art theory since Plato. The history of these debates, which the Renaissance took up again and channeled in more aesthetic than metaphysical directions, has been masterfully summarized by Erwin Panofsky in his celebrated essay of 1924 dedicated to idea. One of the pivotal points of that theory as a whole is the relationship established between the plan or intention of the work (dessein) and the drawing or sketch (dessin) which gives it form. This article argues that modernist painting, although radically abstract and apparently devoted to the triumph of the colour field alone, paradoxically fails to reflect upon itself without recourse to a principle of internal articulation wherein subsist avatars of the old alliance between dessein and dessin. The radical transformation of art's context of production and reception, brought about by the instauration of modernity, introduces however a third term into the equation, design (de-sign), which expresses the fear of a definitive loss: that of the capacity of art to reflect upon the world and provide meaning.
\end{abstract}

... but conception alone is decisive

Clement Greenberg, Art International, 2 octobre 1962

C 'est par l'affirmation de ce principe que le célèbre critique d'art américain Clement Greenberg, dans l'article non moins célèbre qu'il consacrait à l'abstraction du champ coloré1, défendait la production de Barnett Newman, Mark Rothko et Clyfford Still. Cette position surprend un peu, car ce n'est pas habituellement la ligne argumentaire quadopte Greenberg qu'on connaît plus préoccupé d'arrangements formels, pour ne pas dire carrément de style, que d'intentionnalité. En fait, Greenberg a toujours admis la primauté de l'inspiration et l'importance de l'Idée - un concept que recouvre plus ou moins chez lui celui de " contenu " - dans une ouvre d'art. Cette Idée, qu'on ne devra pas confondre avec le sujet d'un tableau, demeure cependant, dans les propres termes du critique, "indefinable, unparaphraseable, undiscussable $»^{2}$. Elle se confond d'ailleurs avec une instance tout aussi volatile : celle de la qualité que seule l'expérience de l'œuvre permet d'appréhender. C'est pourquoi le critique, qui a besoin d'objectiver et de justifier ses jugements, se rabat sur des traits visuellement observables, au risque de se voir taxé de formaliste :

[F]ormalism is stultifying [in] that it begs a large part of the very difficult question as to just what can be sensibly said about works of art. It assumes that " form " and " content " in art can be adequately distinguished for the purposes of discourse. ${ }^{3}$

Ils ne devraient idéalement pas l'être, et on peut suggérer que l'insistance sur la composante conceptuelle, même si elle demeure difficile à saisir, est ici motivée par le contexte : elle serait une réponse aux réticences face à une peinture radicalement abstraite, entre autres celle de Barnett Newman, qui encourt le risque de n'être pas prise au sérieux parce que son exécution paraît à la portée de tous. D'où la déclaration lapidaire, « conception alone is decisive ", dans laquelle on doit entendre un certain non-dit : c'est peut-être facile à réaliser, mais encore fallait-il y penser!

Le présent article émane d'une session de l'AAUC consacrée au concept d'Idea ${ }^{4}$ et placée sous l'égide de l'importante étude qu'en faisait Erwin Panofsky en 19245. Il examine la légitimité d'appliquer à une production moderniste et au discours qui la sous-tend un opérateur théorique associé à l'art du passé, à ses fondements épistémologiques et idéologiques. Comme si une sorte de survivance (ou plutôt une après vie, selon l'acception que Georges Didi-Huberman donne au concept de Nachleben ${ }^{6}$ ) pouvait susciter, dans une métropole américaine $\mathrm{du}$ second $\mathrm{XX}^{\mathrm{e}}$ siècle, une curieuse résurgence des enjeux de la grande tradition classique européenne. On peut rappeler ici, à l'appui d'une telle hypothèse, que la réduction radicale des moyens et la posture résolument autoréférentielle caractérisant la peinture abstraite que défendait Greenberg préparaient l'avènement de propositions artistiques qui, sous l'appellation d'art conceptuel, allaient se réclamer encore plus fortement et plus exclusivement du primat de l'Idée, au point de faire l'économie de l'objet d'art lui-même7. Mais les règles du jeu seraient alors profondément changées, à cause notamment de la critique adressée aux grands médiums traditionnels et à la position dominante qu'avait jusqu'alors occupée la peinture. C'est ainsi que les très vieux débats sur les mérites respectifs du dessin et de la couleur, débats qui traversent la fortune critique d'Idea et qui s'avèrent encore étrangement pertinents pour notre propos, allaient pratiquement sombrer dans l'oubli.

On aura compris que le point de vue adopté ici ne concerne pas l'après-modernisme et les enjeux critiques actuels et que son intention demeure d'abord et avant tout rétrospective. En partant de la peinture abstraite américaine des décennies 1950 et 1960 et de la critique formaliste qui a contribué à sa théorisation autant qu'à sa consécration, il cherche à repérer les derniers avatars d'un questionnement très central dans la pensée esthétique de l'Occident. Les raisons pour lesquelles ce 
questionnement perdure, dans un contexte où l'on se serait attendu à le voir complètement liquidé, sont probablement multiples et incluent une fidélité persistante aux grands médiums classiques qui avaient entretenu cette tradition. La prime de légitimation que l'on pouvait tirer d'une volonté de rattachement au grand art du passé, au moment où l'art étatsunien d'aprèsguerre commençait à s'imposer sur la scène locale et internationale, est aussi à prendre en considération.

Beaucoup pourront s'étonner d'un tel retour à des positions formalistes modernistes que les dernières décennies se sont appliquées à déconstruire avec autant de virulence que le modernisme lui-même s'était attaqué à la tradition. La contestation a depuis longtemps surgi de l'intérieur, à cause de l'arrivée de nouveaux médiums qui ont fait éclater les vieilles spécificités et orienté les questionnements vers les conditions de création, d'exposition et de circulation des œuvres. Elles ont aussi été soutenues, de l'extérieur, par une pratique de l'histoire et de la théorie de l'art plus consciente de ses positions idéologiques, de ses préjugés et de ses exclusions. On a abondamment reproché au formalisme moderniste, avec l'étroitesse de son point de vue et son autoritarisme esthétique, un désintérêt pour le social et le politique qui a très tôt ressemblé à un aveuglement volontaire. Replonger dans le byzantinisme théorique et discursif qui marque cette période, est-il alors utile d'insister, ne signifie pas faire preuve d'innocence et ignorer l'important travail de sape effectué dans le passé récent. L'exercice contribue simplement à mettre en lumière le fait que certaines préoccupations esthétiques ont la vie dure et qu'il est pratiquement impossible d'aborder un médium comme la peinture en se déprenant des vieilles catégories qui, depuis qu'elle s'est imposée dans notre histoire, ont contribué à la penser et à la légitimer.

\section{Panofsky/Greenberg : même combat?}

Il faut néanmoins reconnaittre que l'écart demeure grand entre la peinture de la grande tradition et l'abstraction formaliste moderniste de l'après-Deuxième Guerre. Longue aussi la trajectoire intellectuelle menant Panofsky de Platon au Maniérisme ${ }^{8}$ et la théorisation du High Modernism new-yorkais. Chercher une connexion oblige en effet à ignorer une dimension capitale du concept d'Idea dont l'étude de Panofsky essaie de retracer les développements et les déplacements. Depuis Platon, l'Idée avait toujours eu partie liée avec l'entreprise philosophico-théologique de la connaissance du monde à laquelle l'art souhaitait s'associer. L'histoire d'Idea balise ainsi une tension perpétuelle entre l'empyrée des transcendantaux, qui constitue son premier habitat, et cette réalité sublunaire que l'art s'est donné pour mission de comprendre et d'exprimer en la représentant. Il est vrai qu'à partir de la Renaissance le champ de l'art gagne en autonomie et que la théorie esthétique accorde de plus en plus

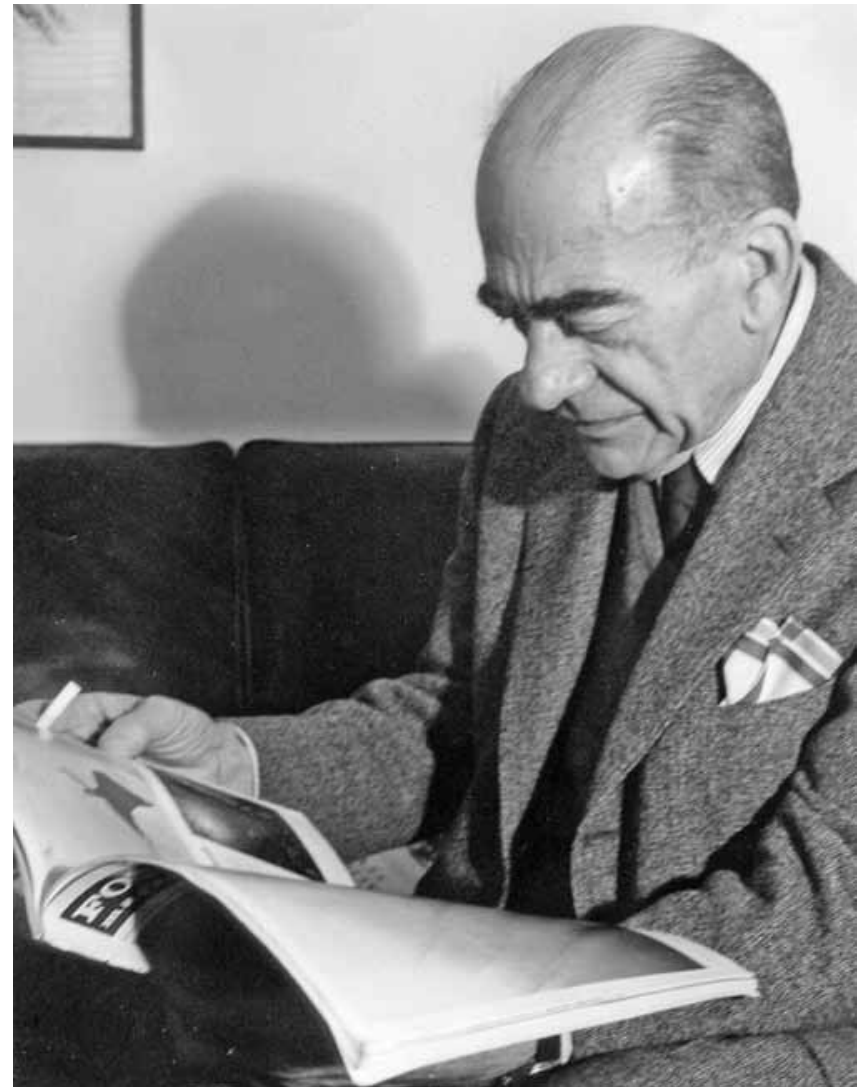

Figure I. Lotte Jacobi, Erwin Panofsky, 1963. Durham, NH, University of New Hampshire (crédit photographique : (c) The Lotte Jacobi Archives. Courtesy of the University of New Hampshire).

d'importance à la médiation du créateur inspiré. Ce qui porte l'éditeur français d'Idea à célébrer, en quatrième de couverture, le caractère foncièrement novateur des développements du concept qu'a connus la Renaissance : "L'origine de la modernité en art est donc contemporaine de ce renversement de sens, bien antérieur à ce qu'indiquent en général les périodisations classiques de l'histoire de l'art. " Prolégomènes à l'art moderne ou pas, reconnaissons que les inventions de l'artiste renaissant concernent d'abord et avant tout l'organisation de figures et de récits imagés, ce que le modernisme a progressivement mis à mal, notamment dans ses vagues successives d'art abstrait, au point qu'on a pu assimiler le nouveau régime à une crise généralisée de la représentation.

L'abstraction semble en effet exprimer la perte de confiance de l'art dans sa capacité à rejoindre et à traduire la réalité. Et le réel, c'est aussi le social. C'est ce que Greenberg devait reconnaître, après Meyer Schapiro, à un moment où il reliait encore l'esthétique et le politique. Revenant sur ce qu'il nomme rétrospectivement sa période trotskyste, le critique américain 


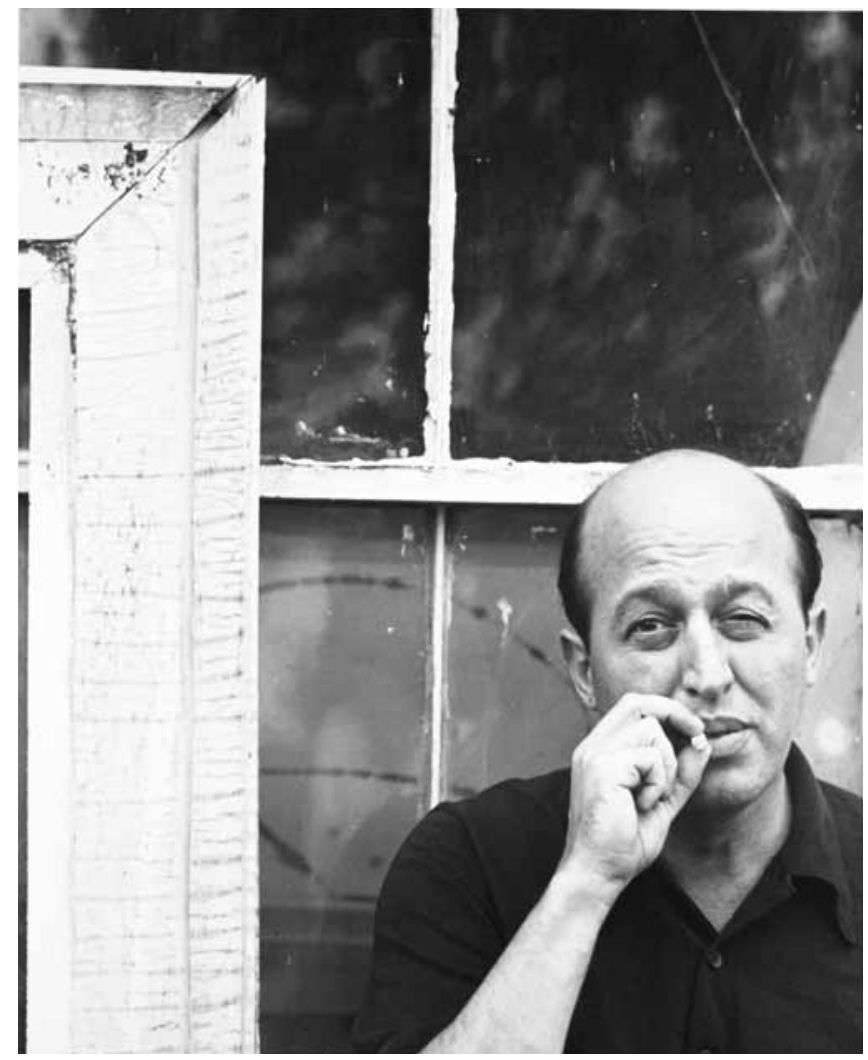

Figure 2. Hans Namuth, Clement Greenberg Outside Jackson Pollock's Studio, 1950. Photographie argentique sur gélatine. Tucson, AZ, University of Arizona (crédit photographique : (1991 Hans Namuth Estate. Courtesy of the Center for Creative Photography, University of Arizona).

convient que l'art d'avant-garde est le produit d'une coupure avec toute forme de collectivité susceptible de lui donner son sens :

A society, as it becomes less and less able, in the course of its development, to justify the inevitability of its particular forms, breaks up the accepted notions upon which artists and writers must depend in large part for communication with their audiences. It becomes difficult to assume anything. ${ }^{9}$

En 1939, au moment où il écrit "Avant Garde and Kitsch " dont est tiré cet extrait, l'art abstrait est pour Greenberg l'expression la plus manifeste de cet état d'aliénation. Cet art s'est retiré de la tâche d'imitation en tant que telle pour imiter, selon sa propre formule, les moyens mêmes de l'imitation ${ }^{10}$.

Une telle position de repli ne correspond pas à ce que Panofsky attendait de l'art. Nous savons qu'il le fit entendre haut et fort dans la querelle épistolaire qui, en 1961, l'opposa au peintre Barnett Newman dans les pages de la revue Art
News. Cet épisode est bien connu ${ }^{11}$ et il ne faut en rappeler ici que la dimension utile à notre propos. Le prétexte de son intervention était le titre d'un tableau de Newman, Vir Heroicus Sublimis (1950-51), reproduit dans la revue et faussement nommé Vir Heroicus Sublimus par suite d'une erreur typographique. Panofsky n'était probablement pas un lecteur assidu d'Art News, mais il avait feuilleté la revue parce qu'on y faisait le compte rendu d'un de ses derniers livres. Pour lui, l'emploi erroné d'un terme de bas latin (sublimus ne veut pas dire sublime, mais, beaucoup plus prosaïquement, physiquement élevé) était symptomatique de la perte de sens qui affectait la production artistique de son temps : «I find it increasingly hard to keep up with contemporary art, particularly with the titles affixed to some of the objects. "12 Objets! Le mot était lâché : confronté à Vir Heroicus Sublimis, Panofsky se trouvait en face d'un artefact qui ne pouvait en aucun cas être porteur d'Idée. C'est pourquoi il considérait son titre prétentieux en plus de le trouver fautif.

Newman avait pourtant mis en place un dispositif qu'il espérait justement porteur d'Idée, une aspiration que sa génération formulait en termes de quête du grand sujet. En plus du titre évocateur, un format ambitieux et une scansion de la surface par des bandes verticales aux espacements longuement médités devaient faire de son tableau une sorte de témoignage philosophique accordé à la sensibilité de l'après-Guerre. Peu d'années auparavant, alors qu'il mettait au point son langage abstrait, l'artiste avait publié quelques essais annonçant que sa peinture visait l'expression du sublime et du tragique. Un de ces essais, rédigé entre 1943 et 1945, s'intitulait "The Plasmic Image " et proposait de rendre l'Idée indissociable de la forme abstraite :

[T] he new painter feels that abstract art is not something to love for itself, but is a language to be used to project important visual ideas. [...] I therefore wish to call the new painting " plasmic » because the plastic elements of the art have been converted into mental plasma. ${ }^{13}$

Newman ambitionnait donc, par une simple configuration de lignes et de couleurs, d'atteindre ce que Panofsky aurait considéré comme le niveau iconologique des significations de l'art ${ }^{14}$. Pour ce dernier, malheureusement, ce à quoi se résume Vir Heroicus Sublimis se situe au registre le plus bas de son modèle interprétatif, le niveau préiconographique ${ }^{15}$, celui où les signes ne font que désigner des objets naturels et où ils n'ont pas encore commencé à faire sens. De toute évidence, Newman visait beaucoup plus haut, très haut en fait! Panofsky ayant suggéré que les artistes modernes devaient se prendre pour Dieu, selon la formule du moine médiéval Aelfric qui plaçait Dieu au-dessus de la grammaire, le peintre américain devait répondre par un acte de foi dans l'art : 
As for the matter of Aelfric, the tenth-century monk had a greater sensitivity for the meaning of the act of creation than does Panofsky. One would think that by now Prof. Panofsky would know the basic fact about a work of art, that for a work of art to be a work of art, it must rise above grammar and syntax - pro gloria Dei.

Clement Greenberg, pas plus que Panofsky ne l'avait fait, ne se laisse cependant prendre aux mots de Newman. Mais ses raisons sont différentes. Le critique s'intéresse peu, nous l'avons déjà évoqué, à l'intentionnalité métaphysique derrière le tableau; ce genre d'Idée relève pour lui de l'indéfinissable et il ne pourrait pas en parler en toute objectivité. Il restera donc sans problème au niveau des configurations de lignes et de couleurs, ce qui va lui permettre d'aborder par le même biais formel les peintres de la première génération de la New York School, ceux qui, comme Newman, Still et Rothko, cherchaient encore à exprimer de grands sujets existentiels, et ceux de la génération subséquente qui, comme Louis, Noland, Olitski et Stella, semblent avoir renoncé à ce type d'ambition. L'Idée qu'il voit à l'œuvre dans l'art de son temps, on le sait bien depuis «Modernist Painting "16, est plutôt liée à une quête d'ordre strictement artistique : celle de l'essence d'un médium qui, par un processus d'épuration trouvant son origine chez Manet, conduit la peinture à se délester de toute Idée qui ne serait pas intrinsèque à sa nature propre (ceci inclut l'usage de sources externes d'inspiration comme la mythologie et l'histoire, la religion et la littérature, mais aussi la contamination plastique d'autres médiums comme la sculpture). L'essence de la peinture, selon la formule lapidaire et relativement simpliste qu'on a abondamment reprochée à Greenberg, se résume dans « Modernist Painting " à une planéité délimitée ${ }^{17}$. Le pessimisme relatif des années 1930 a cédé le pas chez le critique à une attitude beaucoup plus positive : si l'art moderne opère dans le champ étroit de la référence à soi, il ne fait pas qu'exprimer un état d'aliénation sociale. Son gain d'autonomie traduit une indépendance et une autorité nouvelles.

Le traitement que Greenberg réserve à Kant, qu'il salue comme le premier moderniste, est à cet effet révélateur. Pour Panofsky, l'auteur de la Critique de la Faculté de juger était, en plus d'un penseur inspirant, un modèle de dignité humaine. "The History of Art as a Humanistic Discipline "18 rend en effet hommage au philosophe qui, quelques jours avant sa mort, se levait encore à l'arrivée de son médecin parce que, selon ses propres mots, le sens de l'humanité ne l'avait pas encore abandonné. Le Kant de Greenberg est plus qu'un philosophe inspirant; il montre la voie vers la plus haute spécialisation : "Kant used logic to establish the limits of logic, and while he withdrew much from its old jurisdiction, logic was left all the more secure in what there remained of it. "19 Malgré tout cet effort pour penser le modernisme comme un régime à part, Greenberg a néanmoins tendance à minimiser la coupure épistémologique séparant l'art du présent de l'art du passé. Il est de toute évidence moins « innocent " que Panofsky, plongé dans l'archéologie de l'art renaissant pour y suivre les métamorphoses de l'Idée. Le grand promoteur de l'art américain contemporain avait en effet personnellement tout à gagner à prétendre que " ses " peintres se comparaient, en inspiration, en pertinence et en qualité, aux grands maîtres d'autrefois. Il y aurait simplement eu, entre hier et aujourd'hui, un rajustement des priorités :

The limitations that constitute the medium of painting the flat surface, the shape of the support, the properties of the pigment - were treated by the Old Masters as negative factors that could be acknowledged only implicitly or indirectly. Under Modernism, the same limitations came to be regarded as positive factors, and were acknowledged openly. 20

Afin d'appuyer son argument de continuité, Greenberg emprunte un modèle de développement historique à un théoricien appartenant au même milieu de fermentation intellectuelle que Panofsky et qui voulait, comme lui, "rendre compte de la contemplation esthétique par des catégories empruntées à la vie de l'esprit comme producteur de schèmes visuels qui informent la perception de l'œuvre artistique. "21 Il s'agit de Heinrich Wölfflin, dont l'approche formaliste complémentait la démarche iconographique de Panofsky et convenait davantage à Greenberg, puisqu'elle lui offrait toute une série d'oppositions stylistiques binaires lui permettant de traverser la frontière entre l'art figuratif et l'art abstrait. On se rappellera ici que les Principes fondamentaux de l'histoire de l'art de 1915 ignoraient les productions contemporaines pour se concentrer, à l'instar de ce qu'avait fait Panofsky avec son système interprétatif, sur les grandes périodes de l'art du passé. Greenberg prend explicitement le relais de Wölfflin dans un autre de ses articles célèbres, "After Abstract Expressionism "22, où il tente de définir l'apport particulier de Newman, Still et Rothko au projet moderniste dans son ensemble. L'auteur marque plusieurs points dans cet essai. D'abord en renommant expressionnistes abstraits les peintres gestuels de la New York School comme Pollock et de Kooning, il combat l'appellation d'Action Painters 23 qui leur collait à la peau et qui en faisait de véritables existentialistes du pinceau. Selon Greenberg, ces artistes fournissent plutôt à l'histoire de l'art d'Occident une version aboutie du style pictural (du terme original malerische que l'anglais traduit par painterly) baroque qui constituait, avec le style linéaire de la haute Renaissance, un des pôles majeurs de la stylistique wölflinnienne. Si le style linéaire avait déjà triomphé dans l'abstraction dérivée du cubisme, le style pictural était, jusqu'à l'arrivée des Américains, demeuré irrémédiablement compromis avec la figuration. Mais 


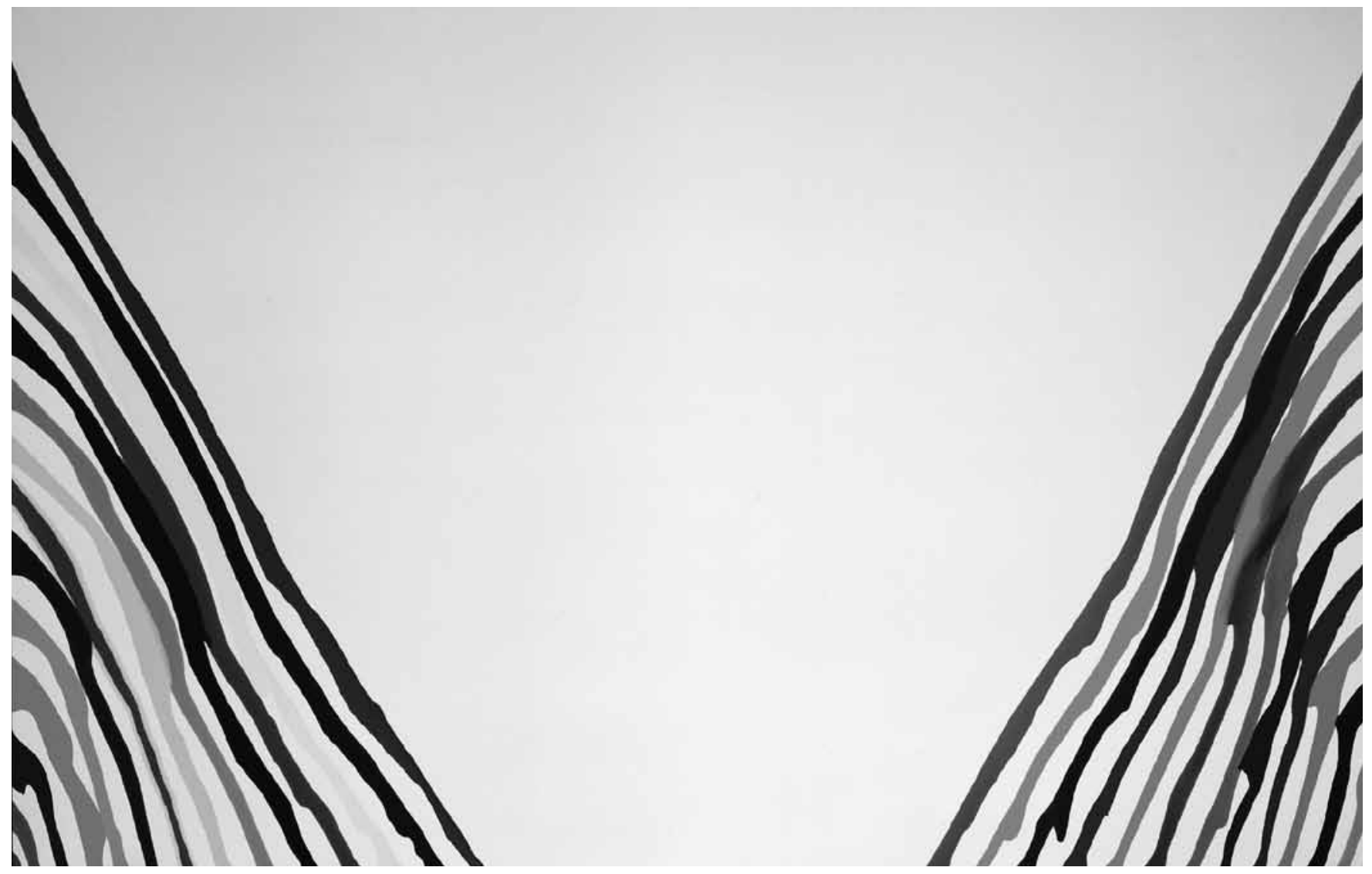

Figure 3. Morris Louis, Beta Lambda. 1961. Polymère synthétique sur toile, 262,6 × $407 \mathrm{~cm}$, New York, The Museum of Modern Art, don de Mme Abner Brenner (crédit photographique : ( The Museum of Modern Art/Licensed by SCALA / Art Resource, NY).

Greenberg réserve la meilleure place à ses peintres du champ coloré. Selon lui, Newman, Rothko et Still transcendent en effet les limites du painterly, dont le traitement s'avère en bout de piste hostile à la couleur, sans renvoyer le balancier vers le style linéaire. L'ouverture nouvelle que manifestent ces peintres correspond à une sorte de synthèse supérieure, à une transcendance pure et simple des polarités wölflinniennes 24 . Hegel n'aurait pas trouvé mieux comme parachèvement de l'histoire!

\section{Dessein/Dessin/Design?}

Ce contexte d'analyse formelle exacerbée remet étrangement à l'honneur la question des rapports dessin/couleur, si importante dans la tradition artistique occidentale; et cette question nous ramène à Idea par de surprenants détours. À la Renaissance, nous l'avons appris entre autres par Panofsky, le concept de disegno constituait un excellent repère pour suivre le travail de l'Idée dans la théorie et dans l'exercice de l'art. Nous n'allons pas refaire ici une histoire qui est déjà bien documentée. Qu'il nous suffise de rappeler que disegno avait à l'origine la double signification de dessein, projet (et c'est sous cet aspect de représentation mentale, de forme élaborée dans l'esprit et dans l'imagination, qu'il est une survivance de l'idée platonicienne) et de dessin (ce qui correspond à l'art du tracé et au sens commun que nous lui donnons encore aujourd'hui). Sa première acception a été bien définie par Federico Zuccaro, peintre et théoricien du Maniérisme, dont l'œuvre maîtresse L'Idea de pittori, scultori ed architetti (ca 1607) la décrit comme une sorte de dessin intérieur ${ }^{25}$. La seconde acception, plus pratique et pédagogique, concerne l'importance accordée à l'enseignement du dessin dans la formation académique des artistes. Même sous cet aspect appliqué, toutefois, le dessin demeurait au plus proche du dessein dans la mesure où il constituait la première matérialisation de l'Idée dans les divers grands médiums qu'étaient la peinture, la sculpture et l'architecture26. Qu'il soit entendu comme projet ou comme tracé, le disegno était cependant inséparable d'une forme de rapport à la nature. Dans la théorie de la Renaissance, l'Idée finit d'ailleurs par céder le pas à l'idéal, sorte de gabarit 
mental supposé permettre à l'artiste de corriger les imperfections du donné sensible et de mieux appréhender le général au cœur du particulier. Elle se présente aussi comme la sœur de l'invention dans la mesure où, une peinture ayant pour but de raconter des histoires, l'artiste doit en imaginer d'avance le scénario visuel avant de l'actualiser sur son support. Il en découle que le dessin comme tracé a d'abord pour fonction de cerner des formes discrètes avant de les regrouper judicieusement dans des ensembles - d'où l'usage courant du terme de circonscriptio, que régulent des traités comme celui d'Alberti :

[J]e désirerais que l'on ne cherche pas à obtenir par la circonscription autre chose que la délimitation des contours, et j'estime qu'il faut s'y exercer assidûment. Ni la composition, ni la réception des lumières ne mériteront de louanges si la circonscription est fautive. Alors que la circonscription à elle seule est déjà très agréable. ${ }^{27}$

On anticipe déjà, à relire l'auteur italien, l'écart important qui s'installe entre la peinture du passé et la peinture moderniste que nous examinons ici. Il n'est en effet plus question, pour l'artiste américain contemporain, d'appréhender le monde en circonscrivant des corps et en les distribuant, dans l'espace fictif de la représentation, pour un maximum d'efficacité décorative et narrative. Même les entités discrètes, géométriques ou informelles, qui peuplaient les peintures abstraites des décennies 1920 et 1930 ont été abandonnées ${ }^{28}$ et, avec elles, les fictions spatiales à quatre, cinq, et $n$ dimensions qui permettaient aux artistes d'avant-garde de projeter leurs utopies visuelles sous forme de grands conflits cosmiques. L'abstraction à l'américaine, qui s'est édifiée sur le terrain du cubo-surréalisme, avait trouvé sa voie en éradiquant à la fois la figure et le décor. Sa première conquête est en effet un mode de composition inédit et sans accent, une organisation all over ${ }^{29}$ qui abolit, avec la dichotomie figure/fond, toute forme de structure hiérarchique. Si l'on rajoute à cette distribution égalitaire et antidramatique l'abandon des effets tactiles qui, selon Greenberg, a constitué l'impératif le plus important de l'autocritique moderniste en peinture, on se demande ce qui peut encore subsister de ce vieux fondement idéel qu'avait été le dessin/dessein. Tout semble en effet emporté par cette recherche de la spécificité du médium peinture : non seulement les contours, mais aussi tous les marqueurs linéaires de la profondeur et des reliefs. Si la cosa mentale de la peinture, c'est la peinture, seul le plan coloré (colour field) doit désormais s'imposer à l'attention.

À revoir l'histoire de cette période, on est frappé par le nombre d'incidents concernant le statut et la fonction du dessin qui semblent devenus de plus en plus précaires. Le critique Harold Rosenberg, grand défenseur de l'abstraction gestuelle comme intervention non préméditée sur la toile vierge, doit justifier que certains des peintres qu'il défend, notamment de
Kooning, puissent faire quand même des croquis : "Il n'y a aucune raison pour qu'une action commencée sur le papier ne se poursuive pas sur une toile. Ou qu'elle se répète à une autre échelle et avec plus de maitrise. Un croquis peut jouer le rôle d'une escarmouche. "30 C'est un dessin de ce même de Kooning qui, avec son accord, devient une œuvre de Robert Rauschenberg sous le titre Erased de Kooning Drawing, 1953, suite à un processus d'oblitération aussi appliqué que systématique.

The drawing was done with a hard line, and it was greasy too, so I had to work very hard on it, using every sort of eraser. But in the end it really worked. I liked the result. It was a legitimate work of art, created by the technique of erasing. 31

Un même coefficient de négativité semble à l'œuvre dans les Black Paintings de Frank Stella, une série de tableaux constitués de bandes de peinture noire séparées par des interstices non peints qui révèlent le support. La critique s'autorise à lire ces espaces en creux comme du dessin et parle de " white pin-striped paintings », ce qui suscite les protestations de l'artiste :

I have never seen a white pin-striped painting [...] there is a distinction between what an artist DOES and what he does NOT do; and however it may lack journalistic appeal, this boundary is precisely the necessary limit without which no work of art can exist. 32

Cette dernière anecdote est révélatrice : les efforts faits par la peinture pour se libérer du dessin et pour se réaliser comme pur espace couleur, une couleur qui, en réitérant la surface de la toile, la fait exister comme tableau, suscitent une réticence à la fois perceptuelle et conceptuelle. Cette dernière témoignerait de la difficulté à déloger le dessin de la position dominante qu'il avait occupée dans toute la tradition artistique d'Occident ${ }^{33}$. Même la révision positive qui permet à Michael Fried, un héritier de Greeenberg, de relire les tableaux de Pollock à la lumière des directions prises par Noland, Olitski et Stella, produit un curieux effet désincarné :

[L]ine, in Pollock's all-over drip paintings of 1947-1950, has been freed at last from the job of describing contours and bounding shapes. [...] Line, in these paintings, is entirely transparent both to the non-illusionistic space it inhabits but does not structure, and to the pulse of pure disembodied energy. [...] Pollock's line bounds and delimits nothing - except, in a sense, eyesight. 34

Ces remarques sont tirées de Three American Painters, un essai important qui sert de catalogue à une exposition tenue au Fogg Art Museum en 1965. Dans les pages qui suivent les remarques sur Pollock, le critique présente les peintres du champ coloré de deuxième génération dont il tente de penser la démarche autrement qu'en termes wölflinniens comme l'avait fait 
Greenberg. Fried insiste tout particulièrement sur les effets purement optiques induits par les vastes plages de couleur qui envahissent de plus en plus les tableaux. Mais il suit son mentor sur un point majeur : la tendance autoréférentielle en train de s'exacerber en peinture n'a rien de programmatique ni d'absolument réducteur : une toile complètement monochrome ne ferait pas nécessairement un bon tableau. L'œuvre risquerait trop de se réduire, littéralement, au simple statut d'objet.

Comment alors distinguer la bonne peinture, celle qui manifeste une conception élevée, de la peinture insignifiante? Paradoxalement, à l'aide du dessin, même si ce dernier se manifeste selon une modalité inédite : le dessin, qui aurait dû disparaître avec la suppression des figures et des fictions spatiales leur permettant de raconter des histoires, le dessin, traditionnellement compromis avec les valeurs tactiles qui sont l'apanage de la sculpture et du bas-relief, maintient une présence structurante d'autant plus obstinée qu'elle se fait discrète. On aurait presque envie de dire aussi " perverse " dans la mesure où, poussé dans ses derniers retranchements par le triomphe de la couleur, le dessin continue d'entretenir des liens de première nécessité avec le dessein. Signalons, rapidement, deux des modalités de sa survivance dans le régime de la peinture pure sur laquelle se penche Michael Fried. La première en fait le résultat d'automatismes réels ou simulés donnant l'impression que le dessin, devenu indissociable de la tache colorée dont il marque la limite, s'est en quelque sorte déployé tout seul, répondant aux lois naturelles de la gravité et de la capillarité. Cette tendance émane des œuvres tardives de Jackson Pollock, produites à l'émail noir imbibé dans la toile brute, transite par les stain paintings d'Helen Frankenthaler et trouve son aboutissement dans les grandes coulées (Veils, Unfurleds) de Morris Louis (fig. 3). Le deuxième usage $\mathrm{du}$ dessin est plus délibéré, mais il dépend exclusivement du rapport établi avec la forme du support qu'il réitère obstinément jusqu'à ce que l'épuisement de la formule appelle le cadre luimême à proposer un dessin intéressant : c'est le shaped canvas que Noland (fig. 4) et Stella (fig. 5) ont bien exploité. Le dessin s'avère ainsi essentiel au maintien d'une syntaxe qui, seule, peut permettre à l'œuvre de faire sens. Fried explique sa position à propos du travail de Noland, dont les formes peintes (désignées depicted shapes et faisant se succéder les cercles, les chevrons et les bandes), établissent un rapport étroit de dépendance avec la découpe de la toile (ce que le critique appelle literal shapes, présentant des profils à géométrie variable) :

[T] he development of pictorial structure in Noland's work is far from arbitrary. [...] In fact, as regards both individual paintings and the development of his work as a whole, structure represents the crux of Noland's response to the crisis of meaning that brought modernism into existence in the first place $[\ldots] .35$

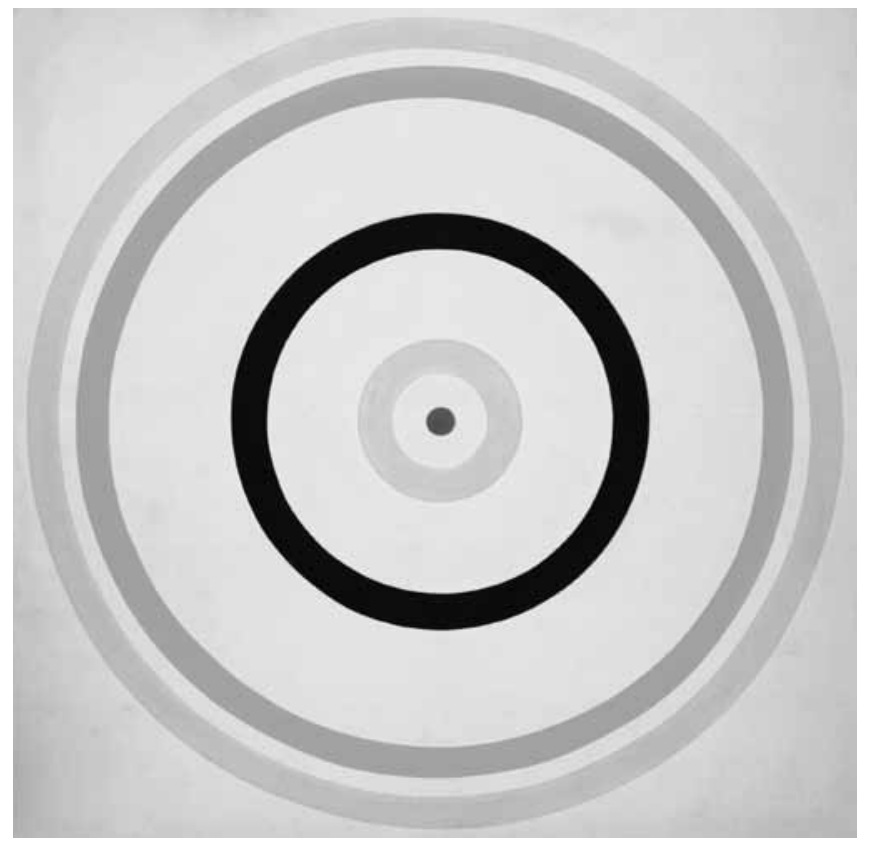

Figure 4. Kenneth Noland, Turnsole, 1961. Polymère synthétique sur toile, $239 \times 239 \mathrm{~cm}$. The Museum of Modern Art, New York, Blanchette Hooker Rockefeller Fund (crédit photographique : () The Museum of Modern Art/Licensed by SCALA / Art Resource, NY).

Pourquoi en serait-on venu à cette situation étrange obligeant une peinture du champ coloré à trouver son sens dans un dessin limite alors même qu'elle est d'abord et avant tout une affirmation chromatique (les reproductions en noir et blanc que nous sommes forcée d'utiliser ici pour l'impression de cet article en offrent une preuve flagrante : présenter les tableaux de Louis et de Noland en version monochrome, c'est littéralement les " défigurer ")? Le critique Max Kozloff a tenté une explication intéressante du phénomène dans un article intitulé fort à propos "Venetian Art and Florentine Criticism "36. Revenant, en 1967, sur la période d'intense production et de théorisation formalistes qui vient de s'écouler, l'auteur essaie d'expliquer la difficulté qu'a posée, aux critiques de son temps, le triomphe absolu de la couleur; cette dernière, en effet, ne serait pas porteuse d'Idée :

[O]f the experience of color, who can say that there is any thought concept that facilitates its rendering in language? Quite obviously, color is unmoored in this discussion because our only means of reference to it is a recollection of the sensation it offers, or possibly an experience similar to the one we are now having. It would seem as if we are reduced to the comparatively primitive operation of naming and recalling. 37 
Michael Fried l'avait explicitement reconnu à propos de Noland : bien que la couleur et la structure aient eu chez lui une égale importance, il était difficile d'appliquer à l'analyse de la première la précision et la rigueur logique que l'on pouvait utiliser pour la seconde ${ }^{38}$. Est-ce alors strictement un problème de réception, les grandes catégories explicatives relevant de l'arsenal mental du critique comme, au temps des pères allemands de notre discipline, elles avaient été l'apanage des historiens d'art eux-mêmes? C'est plus que cela, selon Fried. La conception est bien dans la syntaxe comme on dit du diable qu'il est dans les détails; le fait que la couleur soit plus difficile à caractériser « [...] might easily lead one to overlook the particular source of the kind of statement that can be made about structure and color in Noland's work : namely the fact that structure rather than color bears the brunt of Noland's modernist ambitions. "39 Un argument semblable va soutenir l'attaque en règle que Fried, à peine deux ans plus tard, dirigera contre la sculpture minimale. Dans un autre de ses essais célèbres, "Art and Objecthood " 40 , le critique soutient que les formes primaires proposées par cet art manquent justement de cette articulation syntaxique qui fait de l'œuvre un énoncé signifiant ${ }^{41}$. Les objets spécifiques que propose l'art minimal créent à son avis des situations théâtrales englobant le spectateur et le lieu d'exposition dans une expérience qui, ne trouvant jamais son point d'accomplissement, s'épuise dans la durée. Fried oppose à cet effet de presence, le concept difficilement traduisible de presentness, qu'il assimile à un véritable état de grâce ${ }^{42}$. Comme si l'Idée retrouvait ici une vieille aspiration métaphysique.

Pas pour longtemps! L'Idée peut s'accommoder de bien des régimes et elle va bientôt investir les tautologies de l'art conceptuel, lesquelles ne débouchent sur rien d'autre que sur une Idée de l'art. Mais ce mouvement, qui se situe dans la mouvance du minimalisme, ne fera pas l'économie du contexte et s'intéressera davantage à la fonction de l'art qu'à son autonomie signifiante, surtout une autonomie formulée en termes de pureté du médium. L'étude de l'art conceptuel déborde les limites que nous nous sommes imposées dans cet article. Mais nous trouvons, dans un de ses textes fondateurs, "Art After Philosophy " de Joseph Kosuth, un renversement de perspective auquel nous ne pouvons pas résister, car il va nous permettre de terminer

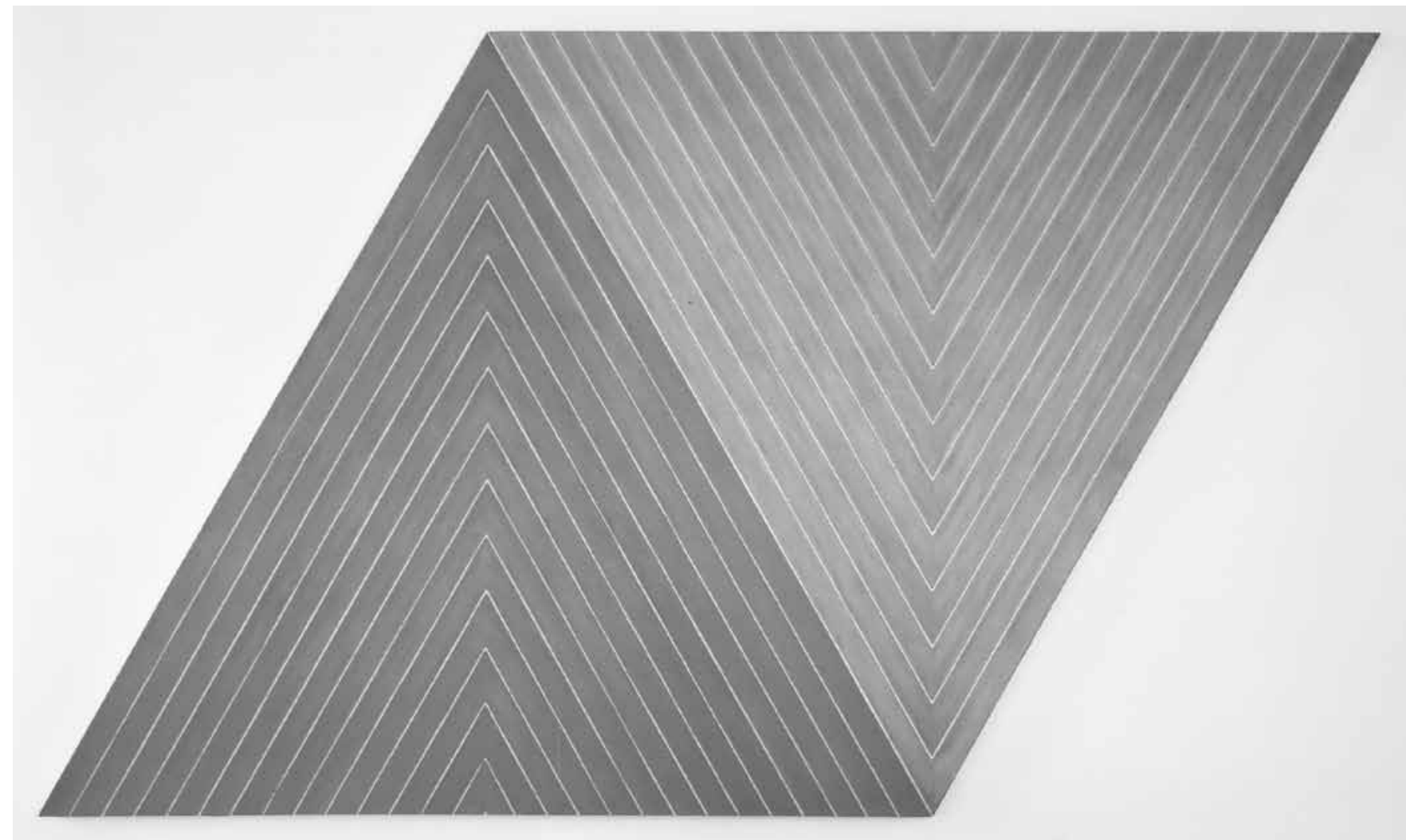

Figure 5. Frank Stella, Valparaiso Flesh and Green, 1963. Peinture métallique sur toile, 198,I × 342,9 ×7,6 cm. Collection de l'artiste Frank Stella (crédit photographique : Art Resource, NY (c) Frank Stella / SODRAC (2012)). 
RACAR XXXVII | Number 2 | 2012

Figure 6. Barnett Newman, Voice of Fire, 1967. Acrylique sur toile, $543,6 \times 243,8 \mathrm{~cm}$. Ottawa, The National Gallery of Canada (crédit photographique: (C) The Barnett Newman Foundation, New York / SODRAC, Montréal (20I2)).

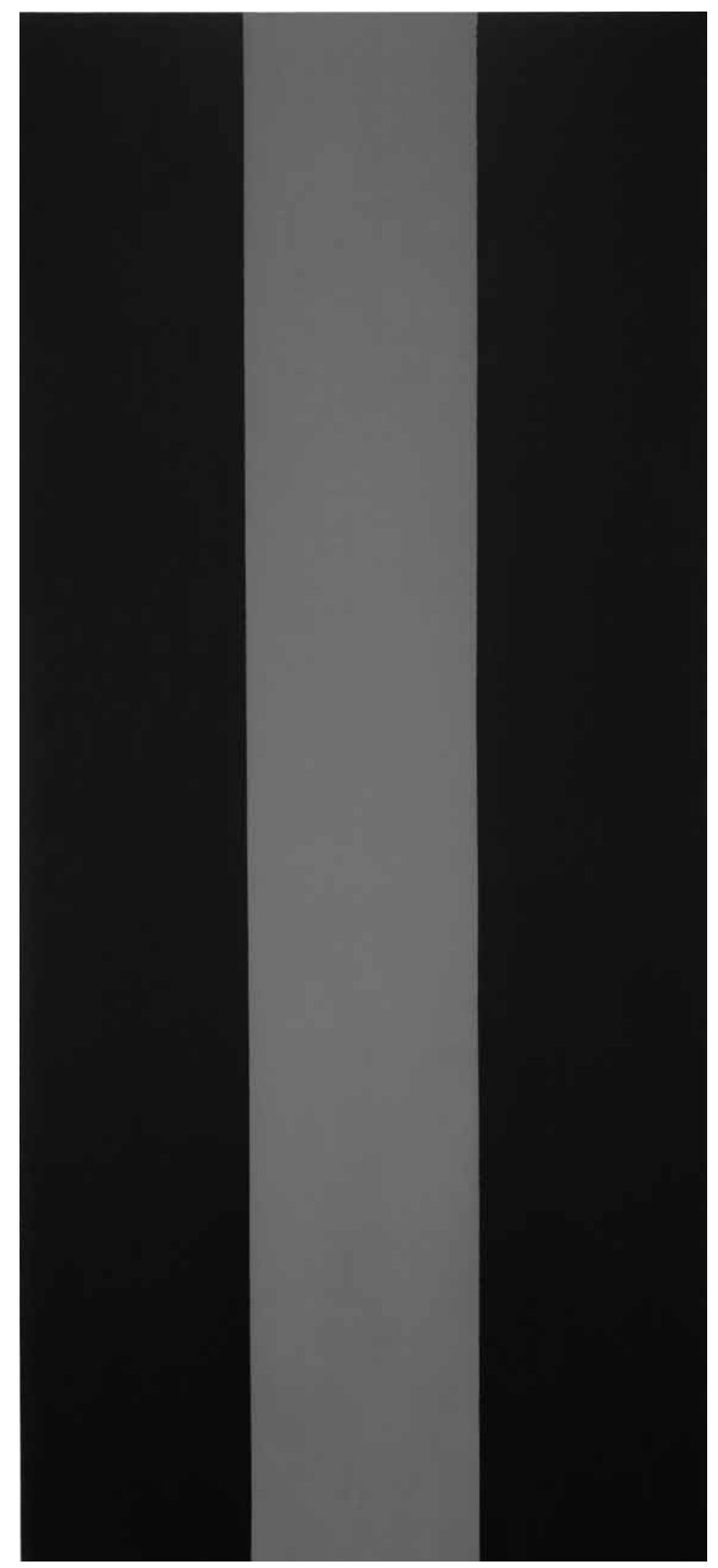




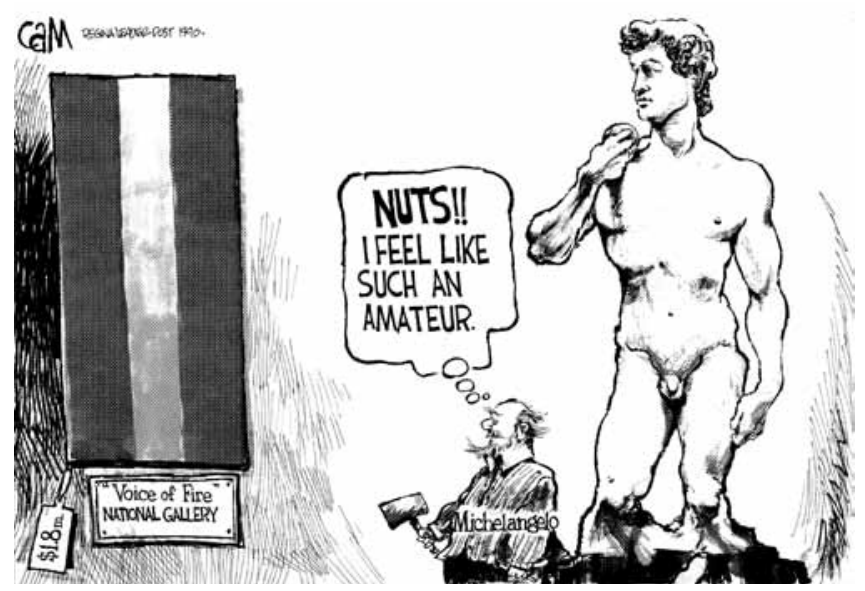

Figure 7. Cameron Cardow, Voice of Fire. Caricature publiée dans The Ottawa Citizen le 25 mars 1990 (crédit photographique : Regina Leader Post).

notre odyssée du concept d'Idea sur une note critique. Pour Kosuth, en effet, la migration de l'Idée vers les propositions de l'art conceptuel fait basculer les peintures du champ coloré du côté des objets ... des beaux objets décoratifs!

Formalist art (painting and sculpture) is the vanguard of decoration, and, strictly speaking, one could reasonably assert that its art condition is so minimal that for all functional purposes it is not art at all but pure exercises in aesthetics. Above all things, Clement Greenberg is the critic of taste. ${ }^{43}$

Comme pour appuyer ce jugement, quelques photographies parues dans la presse artistique montraient l'appartement new-yorkais du critique abondamment pourvu de tableaux du champ coloré qui contribuaient à son décor sophistiqué. Voilà donc apparaître une déclinaison inattendue du disegno, liée cette fois-ci à la perte de sens plutôt qu'à sa plénitude : celle du design, un signe affligé d'un "de " privatif, de-sign, qui le rend caduc en termes d'Idée.

\section{Idéo-logie}

Pour les non-initiés aussi, l'art américain dont il a été question ici est souvent apparu comme une prétentieuse supercherie. Les nombreuses caricatures qui, dans la presse canadienne de 1990, ont commenté l'achat par notre musée national du Voice of Fire de Barnett Newman (fig. 6) au coût de \$ 1.76 million, pointaient pour la plupart dans cette direction; l'immense tableau constitué de trois grandes bandes verticales n'était rien d'autre, pour le contribuable scandalisé, qu'une décoration murale à la facture exorbitante ${ }^{44}$. Un dépliant publicitaire produit par le musée faillit d'ailleurs lui donner raison : la photographie de

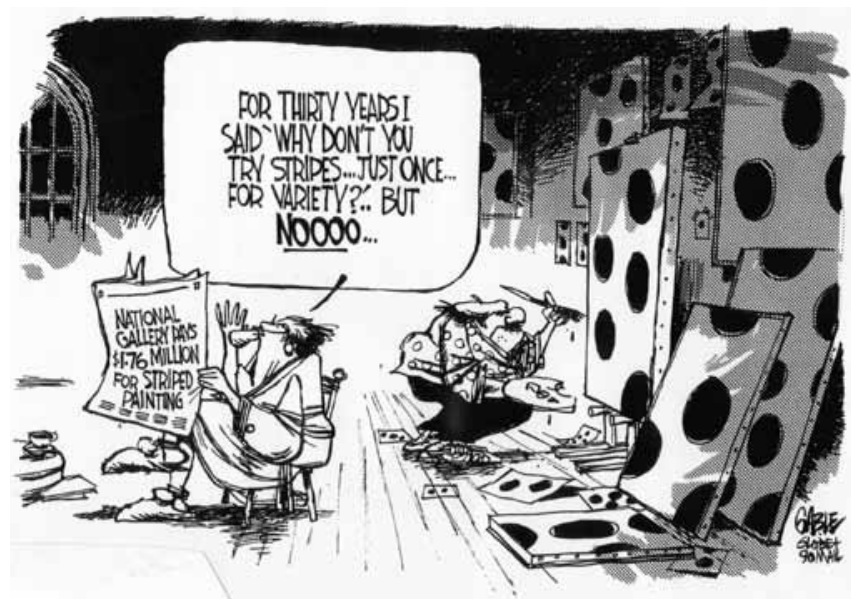

Figure 8. Brian Gable. Caricature éditoriale publiée dans The Globe and Mail le 17 mars 1990 (crédit photographique : Brian Gable/The Globe and Mail).

Voice of Fire avait été inversée, ce que personne n'aurait remarqué n'eut été le logo d'un jeans appartenant à un spectateur en contemplation devant le tableau. Les caricatures (fig. 7) proposaient elles aussi toutes sortes de variations décoratives du Newman, un renversement des bandes à l'horizontale, l'introduction de motifs à pois (fig. 8), histoire de tester leurs chances de succès! Une réplique du Voice of Fire avait été réalisée sur une porte de grange (fig. 9) et une autre sur un toit de maison. Le fait que l'œuvre ait initialement été produite pour le pavillon américain d'Expo 67, à Montréal, et qu'elle se soit dressée comme une bannière immense et visuellement attrayante dans l'espace démesuré du dôme géodésique de Buckminster Fuller n’arrangeait rien. À moins de considérer, et nous voulons terminer sur cette réflexion, qu'elle atteignait un registre iconologique différent de ceux envisagés par l'historien Panofsky et par le critique Greenberg : ce registre d'apparition de l'Idée à un niveau symbolique profond qui rejoint une situation politico-économique donnée a pour nom idéologie.

À Expo 67, l'art américain contemporain était mobilisé pour soutenir la compétition avec la " quincaillerie spatiale » qui s'affichait dans le pavillon russe. La présentation de grandes peintures colorées projetait ainsi l'image d'une Amérique bienveillante, ouverte à toutes les libertés créatrices parce qu'elle ne sentait plus le besoin de prouver sa supériorité technologique. Les abstractions du champ coloré recommençaient donc à parler du social, mais pas sur le ton de l'aliénation qui avait caractérisé les décennies 1930 et 1940 . C’est le genre de constat général que faisait Kozloff dans un article important de 1973 intitulé "American Painting During the Cold War "45. Cherchant les raisons du succès de marché, de la reconnaissance institutionnelle et de l'ascendant sur la scène artistique internationale 


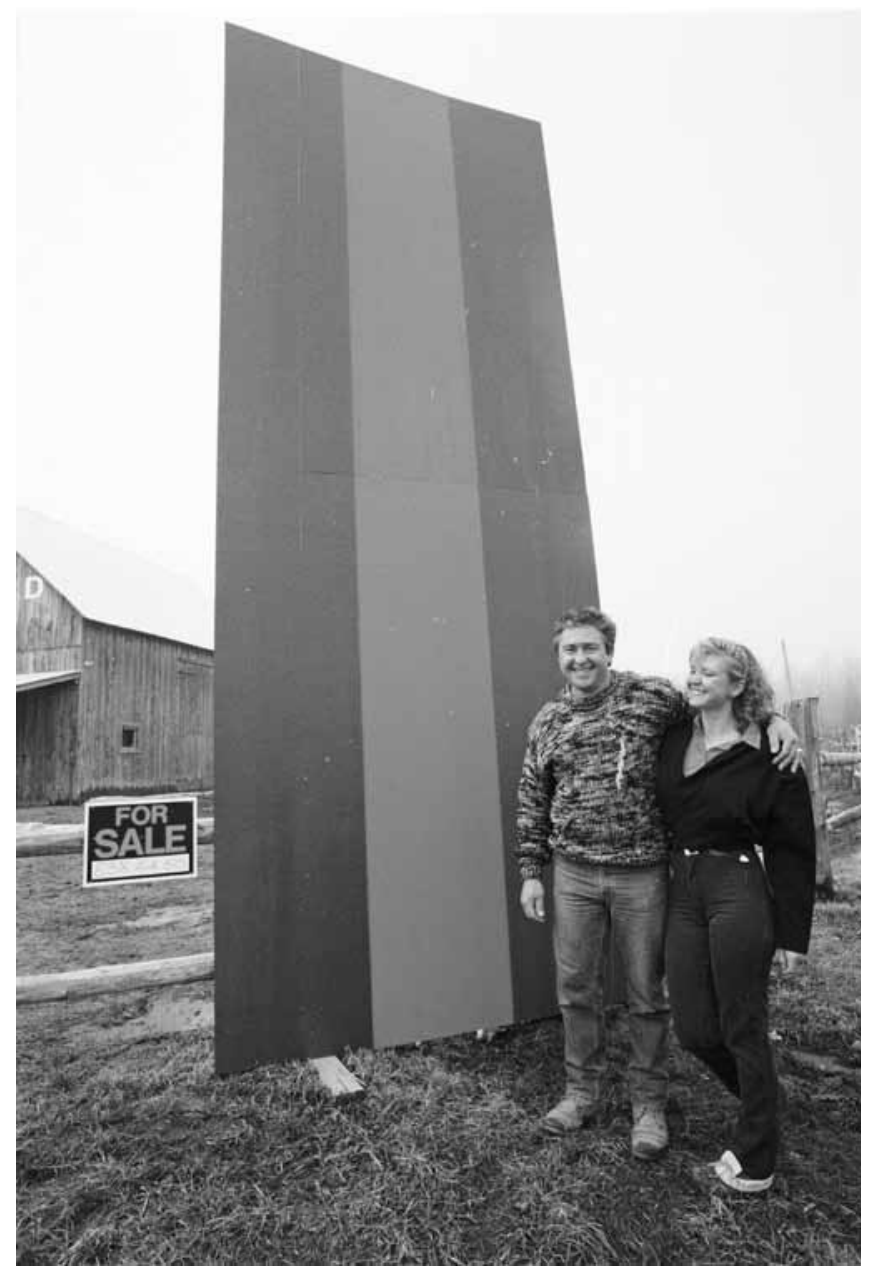

Figure 9. Paul Latour, Voice of the Taxpayer. Photographie publiée dans The Ottawa Citizen le 16 mars 1990 (crédit photographique : Paul Latourl Ottawa Citizen. Reprinted with permission).

qu'avait connus l'abstraction à l'américaine depuis les années 1950, il suggérait que cet art-là manifestait sûrement des connivences profondes avec les valeurs américaines et avec l'impérialisme triomphant qui les portait : "The antiseptic surfacing, the compressed, two-dimensional designing, the optical brilliance, and the gigantism of this art's scale, invoke a far more mundane awe than the sublime. " 46 Le professionnalisme technique manifesté par cet art et son goût de plus en plus accentué pour les productions en séries et pour le renouvellement constant des formules ne sont pas sans rappeler la chaîne de production industrielle dans un régime capitaliste avancé. De cette manière, l'abstraction du champ coloré arrivait encore à transcender le décoratif, mais par des chemins qui ne l'élevaient pas vers la métaphysique.
Nothing interferes with the efficient plotting of its structure - in fact, efficiency itself becomes its pervasive ideal. The strength, sometimes even the passion of this ideal, rescues the best work from the stigma of the decorative, but only to cause it all the more to seem the heraldry of managerial self-respect. ${ }^{47}$

Cette traversée d'un moment fort de la production américaine de l'après-Guerre et des discours la légitimant nous aura en bout de piste appris une chose : il semble que la pratique et la théorie de l'art n'arrivent pas à se passer d'une forme ou l'autre de quête d'Idea. Mais le régime de la modernité, en changeant radicalement les conditions de production et de réception qui avaient présidé à la grande tradition artistique dont Panofsky suit les développements dans son célèbre essai de 1924, transforme la nature du coefficient d'instabilité qui a toujours affecté cette grande question. Ce qui, autrefois, partageait le débat entre les partisans des réalités transcendantales et les partisans des réalités sublunaires demande qu'on cherche aujourd'hui comment des pratiques spécialisées et affligées d'une forte charge d'hermétisme se trouvent quand même, par des voies souvent détournées, interpelées par le social et par l'histoire.

\section{Notes}

1 "What has also been said before, but maybe not emphatically enough, is that the quality of the work of art inheres in its "content" and vice versa. Quality is "content" ». Clement Greenberg, "Complaints of an Art Critic ", Artforum, octobre 1967. Repris dans Clement Greenberg: The Collected Essays and Criticism, éd., John O'Brian, vol. 4, The University of Chicago Press, 1993, p. 269.

2 Ibid.

3 Ibid.

4 Congrès annuel de l'AAUC, Univeristy of Guelph, octobre 2010.

5 Erwin Panofsky, Idea. Contribution à l'histoire du concept de l'ancienne théorie de l'art, trad. Henry Joly, 1re éd. 1924, Paris, Gallimard, Tel, 1989.

6 Georges Didi-Huberman, L'Image survivante. Histoire de l'art et temps des fantômes selon Aby Warburg, Paris, Les Éditions de Minuit, 2002, p. 33.

7 Rappelons qu'en 1973 Gregory Battcock publiait, sous le titre d'Idea Art, une anthologie critique d'articles traitant de l'art récent parus dans Studio International, Arts Magazine et Artforum. On y lit, dans l'introduction, cette définition sommaire que l'on pourrait considérer comme un clin d'œil ironique à Platon : « Works of Idea Art frequently did not actually exist as objects. Rather, they remained ideas... ». Battcock, éd., Idea Art : A Critical Anthology, New York, Dutton, p. 1.

8 Panofsky se permet par exemple de rappeler, dans les commentaires qu'il fait sur Federico Zuccaro et sur son association entre le 
dessin et l'idée, à quel point cette spéculation néo-scolastique est étrangère à la pensée contemporaine. Idea, op. cit., p. 112.

9 Greenberg, "Avant Garde and Kitsch ", Partisan Review, automne 1939. Repris dans Clement Greenberg: The Collected Essays and Criticism, op. cit., vol. I, 1986, p. 6.

10 Ibid., p. 8.

11 On en trouve la transcription intégrale dans Harold Rosenberg, Barnett Newman, New York, Abrams, 1978, p. 244-45. Voir aussi notre article, "Barnett Newman : Thightrope Metaphysics ", dans Bruce Barber et al., éd., Voice of Fire : Art, Rage, Power, and the State, Toronto, University of Toronto Press, 1996, p. 153-65.

12 Rapporté dans Nicole Dubreuil, "Barnett Newman : Tightrope Metaphysics ", op. cit., p. 154." ?

13 Extraits repris dans Thomas B. Hess, Barnett Newman, New York, The Museum of Modern Art, 1971, p. 38.

14 «Intrinsic Meaning or Content. It is apprehended by ascertaining those underlying principles which reveal the basic attitude of a nation, a period, a class, a religious or philosophical persuasionunconsciously qualified by one personality and condensed into one work. " Panofsky, Studies in Iconology: Humanistic Themes in the Art of the Renaissance, 1re éd. 1939, New York, Harper Torchbooks, 1965 , p. 7.

15 Panofsky en donne la définition suivante : "Primary or Natural Subject Matter, subdivided into factual and expressional. It is apprehended by identifying pure forms, that is : certain configurations of line and colour, or certain peculiarly shaped lumps of bronze or stone, as representations of natural objects [...] ». Ibid., p. 5 .

16 Cet article, plusieurs fois remanié et publié, est d'abord paru dans Arts Yearbook en 1961. Nous utilisons la version tirée de Clement Greenberg: The Collected Essays and Criticism, op. cit., vol. 4, p. $85-94$.

17 Ibid., p. 87.

18 Cette anecdote est rapportée dans un essai célèbre, "The History of Art as a Humanistic Discipline ", qui sert de chapitre d'introduction au recueil d'articles de Panofsky, Meaning in the Visual Arts, New York, Doubleday Anchor Books, 1955, p. 1.

19 Greenberg, "Modernist Painting ", dans Clement Greenberg: The Collected Essays and Criticism, op. cit., vol. 4, p. 85.

20 Ibid., p. 86.

21 Jean Molino dans la préface de Panofsky, Idea, op. cit., p. vi.

22 Publié originellement dans Art International, 25 octobre 1962. Dans Clement Greenberg: The Collected Essays and Criticism, op. cit., vol. 4, p. 123.

23 Celle-ci avait été proposée par un rival de Greenberg, le critique Harold Rosenberg, dans un ouvrage de 1959, The Tradition of the New, publié chez Horizon Press. Le concept d'action painting valorisait l'expérience vécue par le peintre au moment de la réalisation de l'œuvre, et ce au détriment du produit fini.

24 "Still, Newman, and Rothko turn away from the painterliness of Abstract Expressionism as though to save the objects of painterliness - color and openness - from painterliness itself. This is why their art could be called a synthesis of painterly and non painterly or, better, a transcending of the differences between the two. " Greenberg, "After Abstract Expressionism ", dans Clement Greenberg: The Collected Essays and Criticism, op. cit., vol. 4, p. 129.

25 Le dessin intérieur (disegno interno) n'est rien d'autre qu' " une forme ou une idée qui réside en notre esprit et qui désigne, avec une explicite clarté, les choses que celui-ci représente. "Zuccaro, rapporté dans Panofsky, Idea, op. cit., p. 107.

26 Voir cette déclaration de Vasari dans Idea, op. cit., p. 80 : « Le des$\sin$, qui est le père de nos trois arts, produit, à partir d'une multiplicité de choses, un jugement universel (giudizio universale) comparable à une forme ou à une idée embrassant toutes les choses de la nature, qui, dans toutes ses proportions, est elle-même entièrement soumise à des règles. "

27 Alberti, De la peinture. De Pictura (1435), 1 re éd., 1992, trad. Jean-Louis Schefer, Paris, Macula, Dédale, 1993, p. 147. Il est intéressant de rappeler ici que la circonscriptio avait joué un rôle fondamental dans les querelles iconoclastes ayant secoué le haut Moyen Âge. Les Antirrhétiques de Nicéphore se portent à la défense des images en arguant que c'est l'humanité du Christ qui rend sa figure circonscriptible : un bel exemple de réconciliation entre la dimension métaphysique de l'Idée et son incarnation dans l'art. Voir Nicéphore, Discours contre les iconoclastes, trad., présentation et notes par Marie-Josée Mondzain-Baudinet, Paris, Klincksieck, 1989 , p. 82.

28 Ce que l'on trouverait entre autres chez Kandinsky et Malévitch.

29 Que le français traduit souvent et laborieusement par l'expression «bord à bord".

30 Harold Rosenberg, La Tradition du nouveau, trad. Anne Marchand, Paris, Les Éditions de Minuit, 1962, p. 26.

31 Anecdote rapportée dans Calvin Tomkins, The Bride and the Bachelors. Five Masters of the Avant-Garde, Duchamp, Tinguely, Cage, Rauschenberg, Cunningham, édition revue et augmentée, New York, The Viking Press, s.d., p. 211.

32 Rapporté dans William S. Rubin, Frank Stella, New York, The Museum of Modern Art, 1970, p. 18.

33 Il y aurait ici tout un chapitre à ouvrir sur les connotations sexuelles de cette primauté et de cette autorité du dessin. On sait que, dans les traités classiques, le dessin a traditionnellement été perçu comme masculin alors que le féminin était réservé à la couleur.

34 Michael Fried, Three American Painters : Kenneth Noland, Jules Olitski, Frank Stella, catalogue d'exposition, Cambridge, MA, Fogg Art Museum, 1965, p. 14.

35 Ibid., p. 24.

36 Max Kozloff, "Venetian Art and Florentine Criticism », Artforum, décembre 1967. Repris dans Kozloff, Renderings : Critical Essays on a Century of Modern Art, New York, Simon and Schuster, Clarion, 1969, p. 321-35. 
37 Ibid., p. 324.

38 "Color tends to be much harder than structure to characterize precisely ". Fried, Three American Painters, op. cit., p. 24.

39 Ibid.

40 Originellement publié dans Artforum, juin 1967. Repris dans Battcock, éd., Minimal Art : A Critical Anthology, New York, Dutton, 1968, p. 116-48.

41 "This preoccupation marks a profound difference between literalist work and modernist painting and sculpture. It is as though one's experience of the latter has no duration - not because one in fact experiences a picture by Noland and Olitski or a sculpture by David Smith or Caro in no time at all, but because at every moment the work itself is wholly manifest. " Fried, " Art and Objecthood", op. cit., p. 145.

42 L'article se termine sur cette déclaration célèbre aux connotations presque religieuses : "Presentness is grace ». Ibid., p. 147.

43 Joseph Kosuth, "Art After Philosophy, I and II ", originellement publié dans Studio International, octobre-novembre 1969. Repris dans Battcock, éd., Idea Art, New York, Dutton 1973, p. 77.

44 Voir, pour une analyse de l'ensemble du dossier de presse, notre article: "Is Less More? The Voice of Fire Controversy ", dans Shelley Hornstein, éd., Capital Culture : A Reader on Modernist Legacies, State Institutions and the Value(s) of Art, Montréal et Toronto, McGill Queen's University Press, p. 97-116.

45 L'article est paru dans Artforum, vol. 11, no 9, mai 1973, p. 43-54. Il correspond à un moment de forte conscientisation politique suscitée, entre autres, par la guerre du Viet Nam.

46 Ibid., p. 54.

47 Ibid. 